\title{
In pursuit of gamma-ray burst progenitors: the identification of a sub-population of rotating Wolf-Rayet stars
}

\author{
J. S. Vink ${ }^{1}$, G. Gräfener ${ }^{1}$, and T. J. Harries ${ }^{2}$ \\ 1 Armagh Observatory, College Hill, Armagh, BT61 9DG, Northern Ireland \\ e-mail: jsv@arm.ac.uk \\ 2 School of Physics and Astronomy, University of Exeter, Stocker Rd, Exeter EX4 4QL, UK \\ Received 3 October 2011 / Accepted 23 November 2011
}

\begin{abstract}
Long-duration gamma-ray bursts (GRBs) involve the most powerful cosmic explosions since the Big Bang. Whilst it has been established that GRBs are related to the death throes of massive stars, the identification of their elusive progenitors has proved challenging. Theoretical modelling suggests that rotating Wolf-Rayet (WR) stars are the best candidates. Wolf-Rayet stars are thought to be in advanced core burning stages, just prior to explosion, but their strong stellar winds shroud their surfaces, preventing a direct measurement of their rotation. Fortunately, linear spectropolarimetry may be used to probe the flattening of their winds because of stellar spin. Spectropolarimetry surveys have shown that the vast majority of WR stars (80\%) have spherically symmetric winds and are therefore rotating slowly, yet a small minority (of 20\%) display a spectropolarimetric signature indicative of rotation. Here we find a highly significant correlation between WR objects that carry the signature of stellar rotation and the small subset of WR stars with ejecta nebulae that have only recently transitioned from a previous red sugergiant or luminous blue variable phase. As these youthful WR stars have yet to spin-down because of mass loss, they are the best candidate GRB progenitors identified to date. When we take recently published WR ejecta nebula numbers (of Stock \& Barlow 2010, MNRAS, 409, 1429), we find that five out of the six line-effect WR stars are surrounded by ejecta nebulae. The statistics imply that the null hypothesis of no correlation between line-effect WR stars and ejecta nebulae can be rejected at the $0.0004 \%$ level. Given that four line-effect and WR ejecta nebula have spectroscopically been confirmed to contain nucleo-synthetic products, we argue that the correlation is both statistically significant and physically convincing. The implication is that we have identified a sub-population of WR stars that fulfils the necessary criteria for making GRBs. Finally, we discuss the potential of identifying GRB progenitors via linear spectropolarimetry with extremely large telescopes.
\end{abstract}

Key words. stars: mass-loss - stars: winds, outflows - stars: Wolf-Rayet - stars: rotation - gamma-ray burst: general

\section{Introduction}

Gamma-ray bursts (GRBs) comprise the most powerful cosmic explosions since the Big Bang. An overwhelming majority of GRBs are of the long and soft variety, which have been associated with stripped-envelope broad-lined supernovae SNe Ic (Galama et al. 1998; Hjorth et al. 2003; Stanek et al. 2003). GRBs represent the deaths of massive stars out to high redshifts, with the current record-holder lying at $z=9.4$ (Cucchiara et al. 2011).

Given the lack of hydrogen and helium in SN Ic spectra, the direct progenitors of SN Ic are widely expected to be strippedenvelope Wolf-Rayet (WR) stars with strong emission lines originating in dense stellar outflows. Even if some models involve binaries of lower mass stars to be the progenitors of H-poor Ibc SNe events, the broad-lined Ic are widely accepted to originate from WR stars (e.g. Modjaz et al. 2011). The most popular model for the production of a GRB involves the collapsar model where a rotating WR core collapses into a black hole. Yet, alternative GRB production models also invoke stellar rotation as the key ingredient for extracting the energy required to produce GRB jets (Woosley 2011).

Despite considerable progress in GRB research over the last decade, the actual identification of GRB progenitors has turned out to be a rather more challenging pursuit. Whilst it is generally expected that GRB progenitors are rotating WR stars, such objects have yet to be identified. The reason for this having been such a challenge is that it is generally not feasible to derive rotation rates from WR spectra. For main-sequence O-type stars it is possible to straightforwardly measure the projected equatorial rotational velocities $(v \sin i)$ via the line-broadening effect of the stellar rotation on photospheric absorption lines, and O-type stars are indeed found to be mostly rotating rapidly (e.g. Howarth et al. 1997). Although angular momentum loss via stellar winds should slow this rapid rotation during massive star evolution, it is natural to assume that some WR stars could still rotate. Unfortunately, because of the nature of the strong winds from WR stars, all lines are in emission, and the traditional absorption-line method cannot be applied to WR stars.

As an alternative to direct methods, one can search for deviations from sphericity of the WR stellar winds as a consequence of stellar rotation. These wind asymmetries would occur on spatial scales that are far too small to be resolved by direct imaging, or even the current generation of interferometers, but linear spectropolarimetry provides an elegant and unambiguous route to assessing wind asphericity. Continuum photons undergo electron scattering as they pass from the thermalization radius to the observer. If the projected scattering geometry is non-circular in the plane of the sky, the scattering introduces a small but measurable net linear polarization. The line photons, 
WR 6
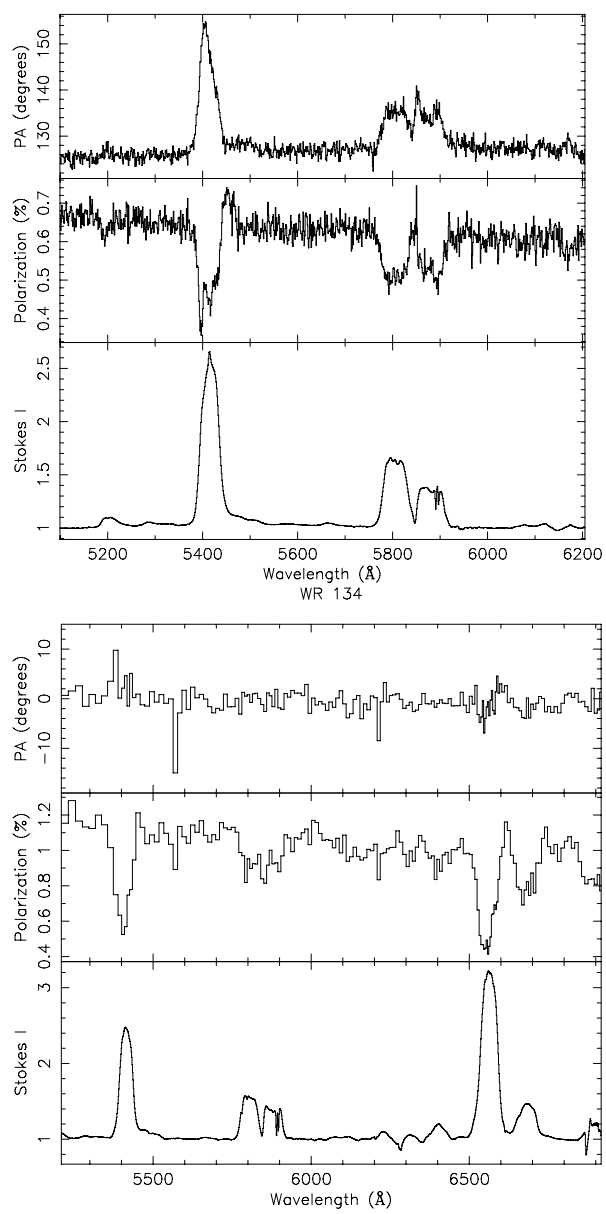

WR 16

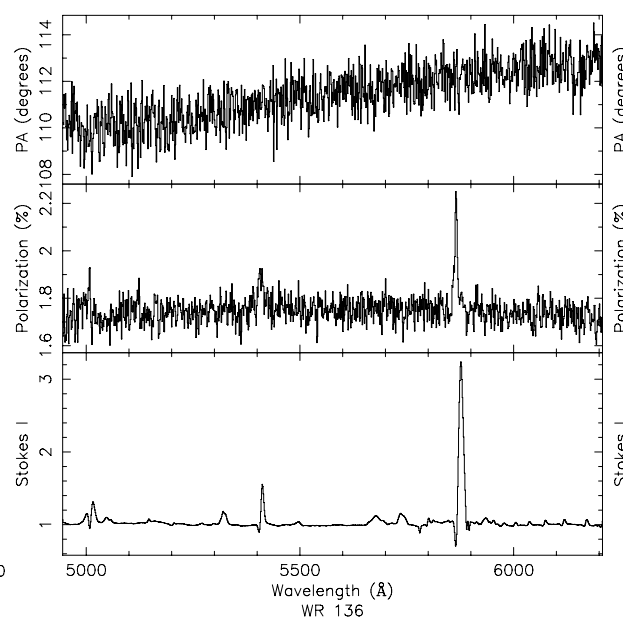

WR 40
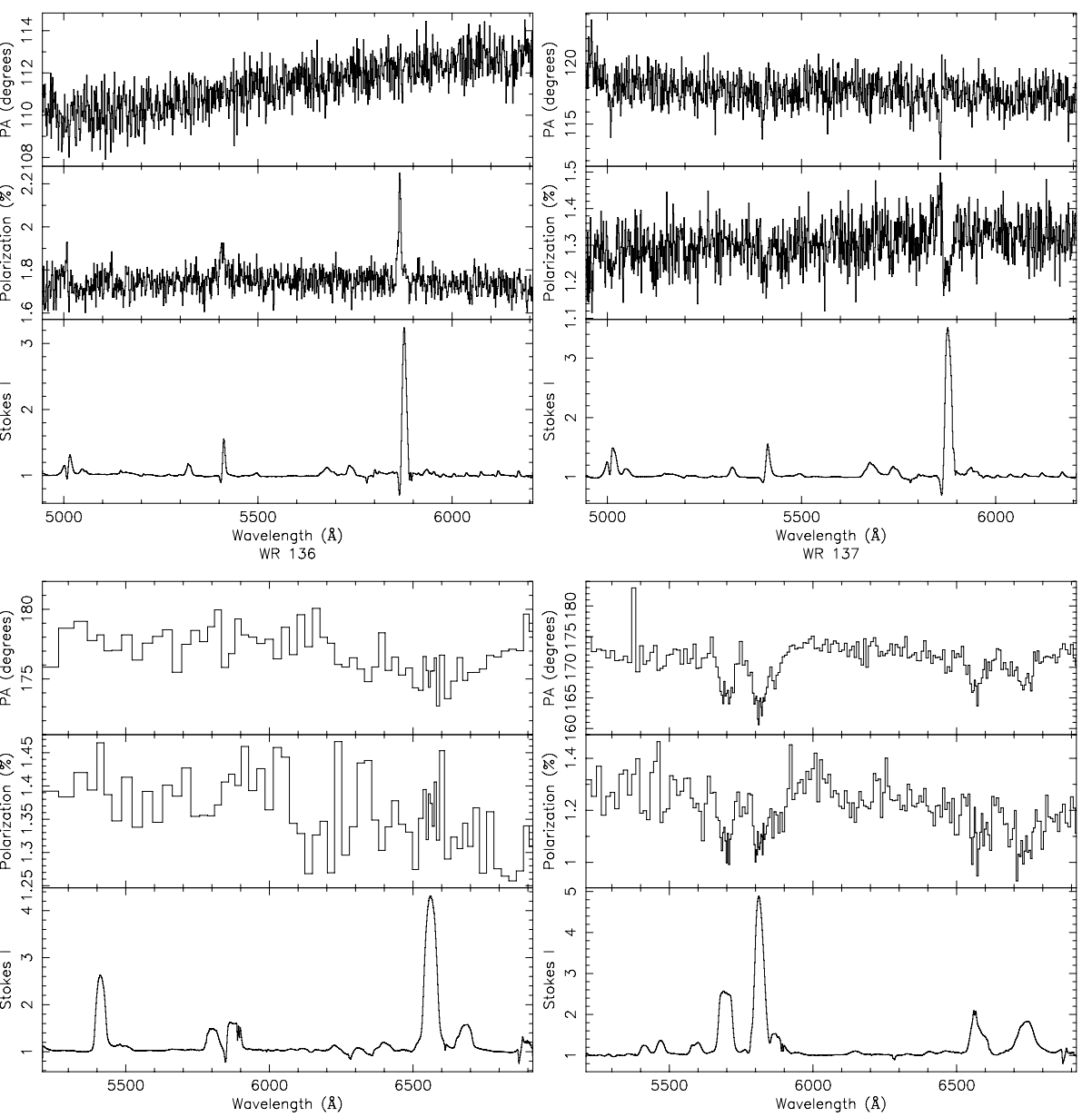

Fig. 1. Galactic line-effect single WR stars (from Table 3 in Harries et al. 1998). The bottom section of each panel shows the normalized intensity spectrum, the middle sections show the polarization magnitude as a percentage, and the top sections show the polarization position angle in degrees. The polarization spectra have been binned to a constant error of 0.05\%. The data for WR 16 and WR 40 are from Schulte-Ladbeck (priv. comm.), the remainder are from Harries et al. (1998).

which are produced at larger radii than the continuum, see a smaller electron-scattering column and are thus less polarized than the continuum. The result is a depolarization at the emission line wavelengths. This effect has been observed in classical Be stars (e.g. Poeckert \& Marlborough 1976), O stars (Harries \& Howarth 1996; Vink et al. 2009), and WR stars.

\section{Spectropolarimetric surveys of WR stars}

More than a decade ago, Harries et al. (1998) investigated the incidence of line effects amongst WR stars. Out of a total sample of 29 Galactic WR stars, six were listed as line-effect objects. In other words, line-effect WR stars were found to be relatively rare, and it was suggested that the "special" property of the line-effect WR stars was related to stellar rotation. Harries et al. assumed that the photometric and spectroscopic variability of the line-effect WR stars was related to the rotation period, which leads to surface rotation rates of the order of $10 \%$ of break-up $\left(\simeq 100 \mathrm{~km} \mathrm{~s}^{-1} \text {; Gräfener et al., in prep. }\right)^{1}$, which may be fast enough to produce significant wind-compression effects according to the models of Ignace et al. (1996).

Close binary stars show intra-binary scattering that can lead to a measurable line-effect, for example CQ Cep

\footnotetext{
1 We note that the stellar core might rotate significantly faster.
}

(Harries \& Hilditch 1997), although the strength of the line effect changes as a function of orbital phase according to the secondharmonic of the period. Therefore the line effect may only be employed as an unambiguous wind diagnostic in WRs that are single or in binary systems where the separation is large enough that intra-binary scattering is negligible (see below).

Harries et al. surveyed the brightest northern hemisphere WR stars and found two single stars (WRs 134 and $137^{2}$ ) that showed the so-called line-effect. The single star WR6 (EZ CMa) also has a strong, variable, line effect (Schulte-Ladbeck et al. 1991; Harries et al. 1999). Other observations of southern hemisphere WRs were presented by Schulte-Ladbeck (1994), who identified two additional single WR stars with a line-effect (WRs 16 and 40). Finally, the star WR136 was reported to have a line effect by Whitney et al. (1988).

In Fig. 1 we show all six single WR stars that have been reported to show a line-effect. WRs 6,16 , and 40 were observed using the RGO spectrograph on the 4-m Anglo-Australian telescope in March 1992. The other objects were observed using the ISIS spectrograph on the 4-m William Herschel Telescope during July 1993. The intensity spectra have been normalized using

2 WR137 is known to be a long (12 yr) period dust producing binary, but the binary separation rules out intra-binary scattering as the polarigenic source (Harries et al. 2000). 
spline fits to interactively defined continuum regions, and the polarization spectra have been binned to a constant error of $0.05 \%$.

Five of the six stars show a substantial depolarization, but WR136 does not. This was noted in Harries et al., but WR 136 was included in the line-effect stars, because a strong photopolarimetric signature was shown in Whitney et al. (1988). For completeness, we have examined the archive spectropolarimetry from Pine Bluff Observatory (PBO). We find the evidence for a line effect in these noisy data to be marginal at best (see Fig. 2). We conclude that WR 136 is a line-effect star with variable continuum polarization, and we urge follow-up spectropolarimetry at high signal-to-noise, performed in a monitoring mode.

Harries et al. (1998) were able to rule out the classical Be star scenario in which all objects are equally asymmetric, and with the observed polarization variation solely because of inclination effects. Although we cannot exclude the possibility that co-rotating interaction regions or other asymmetries could produce some of the linear polarization in certain cases, the most plausible scenario reproducing the observed linear polarization level distribution was shown to be one in which the intrinsic WR polarization levels were biased to low values. This implied that the vast majority $(80 \%)$ of WR stars have spherically symmetric winds indicative of slow rotation, whilst only a small $(20 \%)$ minority have significant intrinsic polarization (of $>0.3 \%$ ). Radiative-transfer modelling demonstrated that these polarization levels were consistent with a wind asymmetry contrast between the low- and high-density mass-loss regions of a factor of 2 to 3 . Harries et al. concluded that the line-effect WR stars are the rotating ones.

A decade later, Vink (2007) considered the line-effect WR stars to constitute the most promising WR population for being GRB progenitors, and therefore studied an analogous sample in the lower metallicity environment of the Large Magellanic Cloud (LMC). Again, line-effect WR stars were found to be rare $(\sim 15 \%)$, with, perhaps surprisingly, no apparent metallicity effect between the Galaxy and the LMC at an Fe content of roughly $50 \%$ that of the Galactic value.

\section{Ejecta nebulae around Wolf-Rayet stars}

Last year, Stock \& Barlow (2010) performed a search for ejecta nebulae around Galactic WR stars inspecting both the northern IPHAS H $\alpha$ survey (Drew et al. 2005) and the southern SHS H $\alpha$ survey (Parker et al. 2005). The fraction of Galactic WR stars with ejecta nebulae was claimed to be low $(\sim 6 \%)^{3}$. A particularly interesting aspect concerning WR ejecta nebulae is that they contain nuclearly processed helium and/or nitrogen enriched material. Ejecta nebulae are thus thought to have formed from strong mass-loss episodes during the prior red supergiant (RSG) or luminous blue variable (LBV) phase, when the outer layers of the star might rotate slowly. If WR stars with ejecta nebulae have only recently transitioned from the RSG/LBV phase, one may possibly expect their central WR stars to be still rotating, before strong and persistent WR winds remove the remaining angular momentum, bringing them to an almost complete standstill.

\footnotetext{
3 We argue that this number is a lower limit. According to our own criteria, the WR ejecta incidence is $23 \%$ (see Gräfener et al., in prep.), making the statistics more conservative, but still highly significant. We note that because the incidence is small, our conclusions are not sensitive to the precise criteria used to identify WR ejecta nebulae.
}
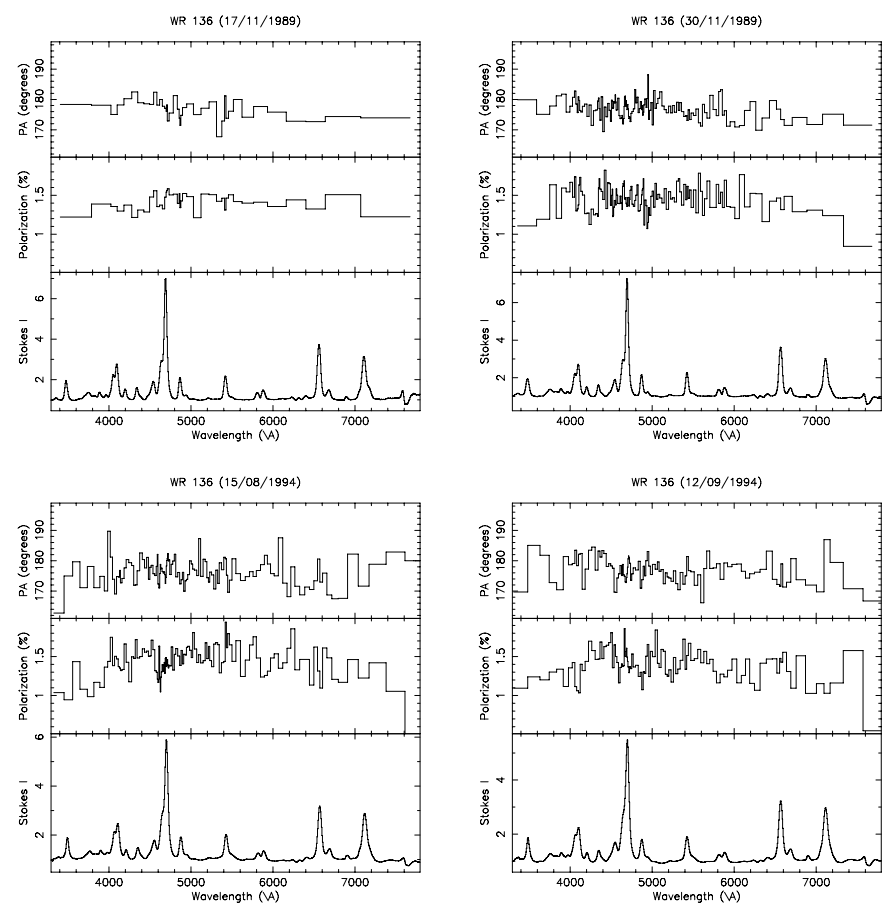

Fig. 2. Spectropolarimetric data on WR 136 taken using the spectropolarimeter at Pine Bluff Observatory in 1989 and 1994. The plots are the same format as Fig. 1 but the data are binned to a constant error of $0.1 \%$.

Table 1. Overlap between the WR ejecta nebula list of Stock \& Barlow (2010) and the line-effect stars according to Table 3 of Harries et al. (1998).

\begin{tabular}{lcc}
\hline \hline WR & Spec.Tp & Reported line-effect WR star? \\
\hline 6 & WN4 & yes \\
16 & WN8 & yes \\
18 & WN4 & no \\
40 & WN8 & yes \\
134 & WN6 & yes \\
136 & WN6 & yes \\
\hline
\end{tabular}

\section{A correlation between WR rotation and youth}

One may ask the question whether the rare population of WR stars that possesses ejecta nebulae and the line-effect stars could be the same objects. The recent and independent list of Galactic WR ejecta nebulae is provided in Table 2 of Stock \& Barlow (2010). The Stock \& Barlow compilation and the Harries et al. (1998) study have just six objects in common, and as many as five of them show a line effect (see Table 1). Taking these numbers (of five out of six) at face value, a binomial test implies that the null hypothesis of no correlation between line-effect WR stars and ejecta nebulae can be rejected at the 0.000004 level $(0.0004 \%)$, i.e. the correlation is highly significant ${ }^{4}$.

Whilst one could possibly argue about morphological details of individual WR nebulae, such as whether a particular nebula does or does not constitute an unambiguous case for being an ejecta nebula (or merely a wind-blown one), given that four line-effect and Stock \& Barlow (2010) WR ejecta nebula have spectroscopically been confirmed to contain nucleo-synthetic

\footnotetext{
${ }^{4}$ If we were to apply our own Galactic WR ejecta nebula incidence rate from Gräfener et al. (in prep.), this number would increase but still be highly significant.
} 
products (see Table 1 in Stock \& Barlow; see also Stock et al. 2011), we argue that the correlation is both statistically significant and physically convincing. Finally, we refer to Gräfener et al. (in prep.) for more detailed discussions, in particular regarding WR nebula morphologies and the relationship to circumstellar absorption features.

\section{Summary and discussion}

We have found a statistically significant correlation between line-effect WR stars that have been postulated to be a rotating sub-population, and WR ejecta nebulae suggesting an evolutionary state just after the RSG/LBV phase. Our line-effect WR subset therefore represents the most promising group of objects fulfiling the criteria to potentially becoming a GRB.

However, GRBs are rare events. Podsiadlowski et al. (2004) estimate that for every broad-lined SNIc-GRB there are at least another 100 non-GRB type Ibc supernovae. This scarcity implies that another property (in addition to rotation rate and evolutionary state) is necessary for a WR star to produce a GRB, for example low iron content (Vink \& de Koter 2005; Yoon \& Langer 2005; Woosley \& Heger 2006; Crowther 2006; Gräfener \& Hamann 2008). This seems very plausible given the significant preference for long GRBs to occur in low-metallicity ${ }^{5}$ galaxies (e.g. Vreeswijk et al. 2004; Fruchter et al. 2006). This appears to be consistent with the tentative upper metallicity limit for GRB progenitors, as suggested by Vink (2007) on the basis of the low line-effect frequency in LMC WR stars, suggesting that the threshold metallicity, where significant differences in WR rotational properties occur, is below $0.5 \mathrm{Z} / \mathrm{Z}_{\odot}$.

In order to discover the most likely population of long GRB progenitors, one would accordingly prefer to find lowmetallicity rotating WR stars. Our results suggest that in principle this could be achieved either by the discovery of lineeffect WR stars in low-metallicity galaxies, or by the search for WR ejecta nebulae in these systems. Given the limited spatial resolution of traditional imaging studies, in practice spectropolarimetry on extremely large (30 m class) telescopes appears to be the most promising avenue for directly pinpointing GRB progenitors - provided these telescopes are equipped with the required polarization optics (Vink 2007, 2010).

We have provided significant additional evidence that the line-effect WR stars are a rotating WR sub-group. This narrows down the group of WR stars that fulfil GRB progenitor criteria, making significant headway towards our final aim of directly pinpointing GRB progenitors.

Acknowledgements. We warmly thank Marilyn Meade from Pine Bluff Observatory and Regina Schulte-Ladbeck from the University of Pittsburgh for providing the spectropolarimetric data on WRs 16, 40, and 136.

\section{References}

Crowther, P. A. 2006, ASPC, 353, 157

Cucchiara, A., Levan, A. J., Fox, D. B., et al. 2011, ApJ, 736, 7

Drew, J. E., Greimel, R., Irwin, M. J., et al. 2005, MNRAS, 362, 753

Fruchter, A. S., Levan, A. J., Strolger, L., et al. 2006, Nature, 441, 463

Galama, T. J., Vreeswijk, P. M., van Paradijs, J., et al. 1998, Nature, 395, 670

Gräfener, G., \& Hamann, W.-R. 2008, A\&A, 482, 945

Harries, T. J., \& Hilditch, R. W. 1997, MNRAS, 291, 544

Harries, T. J., \& Howarth, I. D. 1996, A\&A, 310, 533

Harries, T. J., Hillier, D. J., \& Howarth, I. D. 1998, MNRAS, 296, 1072

Harries, T. J., Howarth, I. D., Schulte-Ladbeck, R. E., \& Hillier, D. J. 1999 MNRAS, 302, 499

Harries, T. J., Babler, B. L., \& Fox, G. K. 2000, A\&A, 361, 273

Hjorth, J., Sollerman, J., Moller, P., et al. 2003, Nature, 423, 847

Howarth, I. D., Siebert, K. W., Hussain, G. A. J., \& Prinja, R. K. 1997, MNRAS, 284,265

Ignace, R., Cassinelli, J. P., \& Bjorkman, J. E. 1996, ApJ, 459, 671

Modjaz, M., Kewley, L., Bloom, J. S., et al. 2011, ApJ, 731, L4

Parker, Q. A., Phillipps, S., Pierce, M. J., et al. 2005, MNRAS, 362, 689

Podsiadlowski, Ph., Mazzali, P. A., Nomoto, K., Lazzati, D., \& Cappellaro, E. 2004, ApJ, 607, L17

Poeckert, R., \& Marlborough, J. M. 1976, ApJ, 206, 182

Puls, J., Springmann, U., \& Lennon, M. 2000, A\&AS, 141, 23

Schulte-Ladbeck, R. E. 1994, ed. A. F. J. Moffat, S. P. Owocki, A. W. Fullerton, \& N. St-Louis (Dordrecht: Kluwer), 347

Schulte-Ladbeck, R. E., Nordsieck, K. H., Taylor, M., et al. 1991, ApJ, 382, 301

Stanek, K. Z., Matheson, T., Garnavich, P. M., et al. 2003, ApJ, 591, 17

Stock, D. J., \& Barlow, M. J. 2010, MNRAS, 409, 1429

Stock, D. J., Barlow, M. J., \& Wesson, R. 2011, MNRAS, accepted [arXiv: 1108.3800$]$

Vink, J. S. 2007, A\&A, 469, 707

Vink, J. S. 2010, Msngr, 14046

Vink, J. S., \& de Koter, A. 2005, A\&A, 442, 587

Vink, J. S., de Koter, A., \& Lamers, H. J. G. L. M. 1999, A\&A, 345, 109

Vink, J. S., de Koter, A., \& Lamers, H. J. G. L. M. 2001, A\&A, 369, 574

Vink, J. S., Davies, B., Harries, T. J., Oudmaijer, R. D., \& Walborn, N. R. 2009, A\&A, 505, 743

Vreeswijk, P. M., Ellison, S. L., Ledoux, C., et al. 2004, A\&A, 419, 927

Whitney, B. A., Schulte-Ladbeck, R. E., Aspin, C., et al. 1988, BAAS, 20, 1013

Woosley, S. E. 2011 [arXiv: 1105.4193]

Woosley, S. E., \& Heger, A. 2006, ApJ, 637, 914

Yoon, S.-C., \& Langer, N. 2005, A\&A, 443, 643

\footnotetext{
5 Note however that these metallicity measurements involve oxygen rather than iron (Vink et al. 2001). For a discussion of the physics of Fe versus CNO line-driving see Vink et al. (1999) and Puls et al. (2000).
} 